\title{
Structure of Stable Sand Piles Model
}

\author{
Phan Thi Ha Duong ${ }^{1,2}$ and Tran Thi Thu Huong ${ }^{2}$ \\ ${ }^{1}$ LIAFA, Universite Paris 7 - 2, place Jussieu, 75005 Paris, Fanrance \\ ${ }^{2}$ Institute of Mathematics, 18 Hoang Quoc Viet, Hanoi, VietNam. \\ phan@liafa.jussieu.fr, tthtran@math.ac.vn
}

\begin{abstract}
In this paper we study a variant of the Sand Piles Model, where the evolution rule consists of the falling down of one grain to a random column and an avalanche to reach a stable configuration. We prove that the infinite set of all stable configurations have a lattice structure which is a sublattice of Young lattice. At the end, based on a discussion about avalanches, we construct a generating tree of this model and show its strongtly recursive structure.
\end{abstract}

Keywords: Sand Piles Model, lattice, Young lattice, generating tree

\section{Introduction}

The Sand Piles Model (SPM) was introduced in 1987 by Bak, Tang and Wiesenfeld as a sample model of the Self organized criticality (SOC) phenomena (5). The authors simulated the behaviour of a sand pile which builds up when sand is dropped on a line. A configuration was modelled as a sequence of columns consisting of cubic sand grains. Addition of new grains is simply performed by selecting a column at random and increasing its height by one. If the column then exceeds its right neighbours in height more than some threshold, it will give some grains to its nearest neighbours. A cascade process sets in until all height differences are below the threshold. The size of such an avalanche is defined as the number of sand grains sliding as a result of a single grain of sand being added to the pile.

This model was investigated in many works in physics and combinatorics $(1 ; 2 ; 4 ; 6)$. By fixing the total number of sand grains, and regarding only avalanches, Goles et al. proved the lattice structure of the space of all reachable configurations (2). After that, by studying the system consisting of avalanches caused by the addition one grain at the first column, Latapy et al. showed the recursive structure of the space of configurations (4). In these models, the threshold is chosen as 2 , so that for each configuration the heights of columns form a decreasing sequence from left to right, and so that these configurations are represented by an integer partitions.

In this paper, we study the original model in keeping the fact that each configuration is still a decreasing sequence. The first configuration has no sand grains, and it is a stable configuration. At each step, one column is selected at random with the condition that its height is smaller than its left neighbours's height. We add one grain to this selected column. After that, if its height is greater than its right neighbours's height by at least two, the system creates an avalanche to reach a new stable configuration. The model of all possible stable configurations, when the total number of sand grains varies from 0 to $\infty$, is called Stable Sand Piles Model and denoted by SSPM.

We observe that by applying the evolution rule to a stable configuration, one can obtain different new stable configurations, they depend on the random column where the grain is added. However, for two different selected columns, one can get the same new stable configuration. For example, if we add one grain to column 4 or to column 2 of the stable configuration $(5,4,3,2,2,1)$, we obtain the same new stable configuration $(5,4,3,3,2,1)$. Notice that in the first case, the new stable configuration is obtained just after adding one grain at column 4 (without creating avalanche), and in the second case, the new stable configuration is obtained after adding one grain at column 2 and an avalanche of size 2 .

In the next section, we investigate the problem of properties of the space of all stable configurations which has an order structure. We show that it is in a bijection with the poset of all strict partitions, both together are sublattice of the well known Young lattice. In section 3, we discuss about the different sizes of avalanches to reach the same stable configuration. Due from shortest chains of avalanches, we introduce a generating tree of SSPM, which has a strongly recursive structure and which can be coded by a line of recursive subtrees.

Before studying properties of $S S P M$, let us give here some preliminary definitions. 
Definition 1 (i) A sequence of positive integers $a=\left(a_{1}, \ldots, a_{k}\right)$ is called a partition of a given integer $n$ if it is decreasing and if $\sum_{i=1}^{k} a_{i}=n$. We call $a_{i}$ part of partition $a$; $n$ weight of a, and write $w(a)=n$; and $k$ length of $a$, and write $l(a)=k$.

(ii) A smooth partition is a partition such that all differences between two consecutive parts are at most 1.

(iii) A strict partition is a partition such that all parts are different.

(iv) Young's lattice is the lattice of all partitions ordered by containment, i.e., $a \leq b$ if and only if $a_{i} \geq b_{i}$ for all $i=1,2, \ldots, \min \{l(a), l(b)\}(7)$.

\section{Characterization of $S S P M$}

We first represent $S S P M$ as a mathematical model. It is clear to see that a stable configuration can be represented by a smooth partition of its total number of sand grains. Hence Stable Sand Piles Model (SSPM) is a discrete dynamical model of smooth partitions such that:

- The initial smooth partition is $(0)$.

- Evolution rule: for a smooth partition $a=\left(a_{1}, \ldots, a_{k}\right), a_{i}$ can increase by one grain if $a_{i}<a_{i-1}$. And if $a_{i}+1 \geq a_{i+1}+2$, this grain can slide down to a new column $i+j, j \geq 1$, until the obtained partition is smooth.

Let us now define the cover relation: a smooth partition $a$ of $n$ covers a smooth partition $b$ of $n+1$ if $b$ can be obtained from $a$ by applying the evolution rule. It is evident that the reflexive and transitive closure of this relation is an order relation. We denote it by $\leq_{S S P M}$.

We first establish the equivalence between stable configurations and smooth partitions.

Proposition 1 The Stable Sand Piles Model generates all smooth partitions.

The infinite set of all smooth partitions equipped with the order $\leq_{S S P M}$ is called the poset Stable Sand Piles Model, and denoted also by SSPM.

Proposition 2 In the SSPM, an element a covers another element $b$ if and only if $b$ can be obtained from a by adding one grain at some column.

The order relation $\leq_{S S P M}$ can be characterized as follows:

Corollary 1 The poset SSPM is ordered by containment.

While consider SPM, one can take a threshold's value different from 2. In the case this value is equal to 3 , one obtains configurations coded by strict partitions. In fact, for a configuration $a=\left(a_{1}, \ldots, a_{k}\right)$, one grain can fall down from $a_{i}$ to $a_{i+1}$ if $a_{i}-a_{i+1} \geq 3$, so after the falling of one grain, $a_{i}$ is still strictly greater than $a_{i+1}$.

Hence, we introduce a model which generates the set of all strict partitions as follows:

- The first element is (0) (by convention, (0) is a strict partition).

- Evolution rule: a part $a_{i}$ of a strict partition $\left(a_{1}, \ldots, a_{k}\right)$ can increases by one with the condition that the obtained partition is still a strict partition.

Similarly to Proposition 1, we can show that this model generates all strict partitions. We define the order $\leq_{\text {strict }}$ between strict partitions: $b \leq_{\text {strict }} a$ if $b$ can be obtained from a by applying a sequence of evolution rule. The infinite set of all strict partitions equipped with this order is called the poset Strict and denoted by Strict. We have the following result on structure of Strict:

Lemma 1 The poset Strict is ordered by containment and it is a sublattice of Young lattice.

Before entering the main theorem of this section we recall some definitions. Let $a=\left(a_{1}, a_{2}, \ldots, a_{k}\right)$ be an integer partition, the dual mapping $\varphi$ is defined by : $\varphi(a)=a^{*}$ is a $l$-tuples $\left(b_{1}, b_{2}, \ldots, b_{l}\right)$ where $b_{i}$ is the number of $a_{j}$ such that $a_{j} \geq i$. One can show that $\varphi$ defines a bijection from SSPM to Strict. Morever for two partitions $a$ and $b, a=b^{\downarrow i}$ if and only if $a^{*}=b^{* \downarrow j}$, where $j=b_{i}$. Then, by virtue of Lemma 1 and Corollary 1 $\varphi$ preserves order relation between SSPM and Strict, so it is a lattice isomorphism. Therefore, we can now state the following result:

Theorem 1 The poset SSPM is a sublattice of Young lattice, and it is in a lattice - isomorphism with the lattice Strict. 


\section{Avalanches and generating tree}

In the first section, we have noted that there are probably different sequences of applying evolution rules from a stable configuration $a$ to reach another stable configuration $b$. In fact, if $b$ is smaller than $a$ by $S S P M$ order, every such sequences have the same number of evolutions, but the number of avalanches sizes may be quite different. So the total time to reach a given stable configuration depends on the chosen columns in which evolution rule is applied. We have some ideas to compute maximal length of sequence to reach a given stable configuration, but the proof is not comlete yet. On the other hand, we show that minimal length sequences consist of evolutions without avalanches, that means every grain fall down directly in its final position.

Theorem 2 The minimal length of sequence to reach a smooth partition of weight $n$ is $n$.

Based on these sequences, we construct an infinite tree which generates all smooth partitions and an infinite tree which generates all strict partitions.

Definition 2 The binary Tree of Strict is defined affollows: The root is the strict parition (0). Each strict partition a has a left child $a^{\downarrow 1}$. Morever, if a begins with a step (a decreasing subsequence where all height differences are equal to 1) of length $i$ and $a_{i}-a_{i+1}=2$ then a has a right child $a^{\downarrow i+1}$.

Definition 3 The binary Tree of SSPM is defined as follows: The root is the smooth parition (0). Each smooth partition a has a left child $a \cup 1$. Morever, if $a^{*}$ begins with a step of length $i$ and $a_{i}^{*}-a_{i+1}^{*}=2$ then a has a right child $a^{\downarrow i+1}$.

Theorem 3 The Tree of Strict is a spanning tree of the graph of lattice Strict. The Tree of SSPM is a spanning tree of the graph of lattice SSPM. And these two trees are isomorphic.

Recall that for a lattice $\left(L, \leq_{L}\right)$, we say graph of $L$ the graph whose vertices are elements of $L$ and whose edges are cover relations of $L$. We label each edge $a \rightarrow a^{\downarrow_{j}}$ of these tree by $j$. The spanning tree of the graph of lattice Strict defined as above is in fact the same spanning tree defined by Le and Phan in (3). This tree has a strong recursive structure as we present in the following. We call $X_{k}$ subtree any left subtree of an element of the form $(p, p-1, \ldots, p-k+1, p-k-1, \ldots)$. Moreover, we define $X_{0}$ as a simple node. The next proposition shows that all the $X_{k}$ subtrees are isomorphic.

Proposition $3 A X_{k}$ subtree, with $k \geq 1$, is composed by a chain of $k+1$ nodes (the rightmost chain) whose edges are labeled $1,2, \ldots, k$ and whose $i$-th node having an out going edge labeled with 1 to a $X_{i}$ subtree for all $i$ between 1 and $k$.

This recursive structure and the above propositions allows us to give a compact representation of Tree of Strict by a chain.

Theorem 4 The tree Tree of Strict can be represented by the infinite chain

()$, 1,2,21,31,32,321, \ldots,(n-1, n-2, \ldots, 1),(n, n-2, \ldots, 2,1), \ldots$,

$(n, n-1, \ldots, 3,2),(n, n-1, \ldots, 3,2,1), \ldots$ with corresponding edges

$1,1,2,1,2,3, \ldots, 1,2, \ldots, n, \ldots$; each node before an edge $k$ having an out going edge labeled with 1 to the root of a $X_{k-1}$ subtree.

Morever, we can prove a stronger property of each subtree in this chain:

Theorem 5 The subtree (of the form $(k, k-1, \ldots 2,1) \stackrel{1}{\longrightarrow} X_{k}$ ) of Tree of Strict contains exactly the strict partitions of length $k$.

Due from the bijection between strict partitions and smooth partitions, we have similar results for Tree of SSPM.

\section{Appendix}

Sketch of proof : (of Proposition 1): We prove by reccurence on the total number of grains. Suppose that after $n$ steps, the system generates all smooth partitions of $n$, we show that an arbitrary smooth partition $\left(a_{1}, \ldots, a_{k}\right)$ of $n+1$ can be obtained. Let $j$ be the first index such that $a_{j-1}=a_{j}, a_{j}=a_{j+1}+1$ (if such an index does not exists, we take $j=1$ ). Let $b$ be the partition of $n$ defined by $b_{i}=a_{i}$ for all $i \neq j$ and $b_{j}=a_{j}-1$. It is clear that $b$ is a smooth partition of $n$ and $a$ is obtained from $b$ by applying evolution rule at position $j . \diamond$

Sketch of proof : (of Proposition 2): By definition, $b$ is covered by $a$ if $b$ can be obtained from $a$ by applying evolution rule. Suppose that this evolution rule consists of one adding a grain at position $i$, and an 
avalanche of size $j$. One can show that $b$ can be obtained directly from $a$ by adding directly one grain to column $i+j$. $\diamond$

Sketch of proof : (of Lemma 1): Let $a$ and $b$ be two partitions in Young lattice, and let $k=$ $\max (l(a), l(b))$. Recall that the formulas for $\inf (a, b)$ and $\sup (a, b)$ are:

$$
\begin{aligned}
& \sup (a, b)=\left(\min \left\{a_{1}, b_{1}\right\}, \min \left\{a_{2}, b_{2}\right\}, \ldots, \min \left\{a_{k}, b_{k}\right\}\right) \text { and } \\
& \inf (a, b)=\left(\max \left\{a_{1}, b_{1}\right\}, \max \left\{a_{2}, b_{2}\right\}, \ldots, \max \left\{a_{k}, b_{k}\right\}\right) .
\end{aligned}
$$

One can check that if $a$ and $b$ are strict partitions then in $f(a, b)$ and $\sup (a, b)$ are also. $\diamond$

Sketch of proof : (of Theorem 2): Using the idea in the proof of Proposition 1], one can construct an inverse chain from a given smooth partition come back to the initial smooth partition (0). Each evolution is of the form $a=b^{\downarrow j}$ where $j$ being the first index such that $a_{j-1}=a_{j}, a_{j}=a_{j+1}+1$ (if such an index does not exists, we take $j=1$ ). $\diamond$

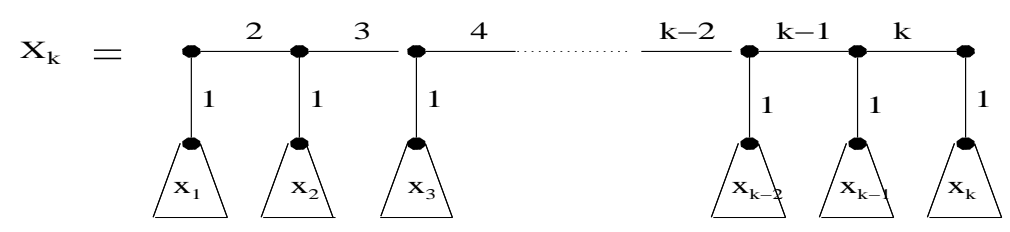

Fig. 1: Self-referencing structure of $X_{k}$ subtrees.

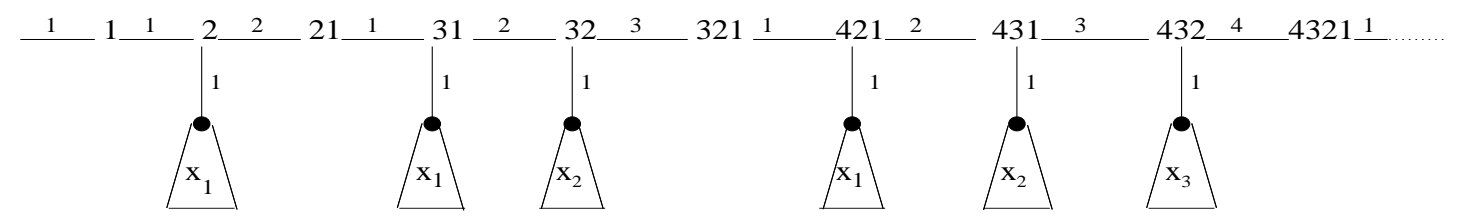

Fig. 2: Representation of Tree of Strict as a chain.

\section{References}

[1] E. Goles and M.A. Kiwi. Games on line graphes and sand piles. Theoret. Comput. Sci., 115:321-349, 1993.

[2] E. Goles, M. Morvan, and H.D. Phan. Sandpiles and order structure of integer partitions. Discrete Appl. Math., 117:51-64, 2002.

[3] Le Minh Ha and Phan Ha Duong. Strict partitions and discrete dynamical systems. FPSAC'04. Vancauver, Canada, page 12 pages, 2004.

[4] M. Latapy, R. Mataci, M. Morvan, and H.D. Phan. Structure of some sand piles model. Theoret. Comput. Sci, 262:525-556, 2001.

[5] P.Bak, C. Tang, and K. Wiesenfeld. Self-organized criticality: An explanation of 1/f noise. Physics Rewiew Latters, (59):381, 1987.

[6] J. Spencer. Balancing vectors in the max norm. Combinatorica, (6):55-65, 1986.

[7] Richard P. Stanley. Enumerative combinatorics. Vol. 2. Cambridge University Press, Cambridge, 1999. 